\title{
Biofunctional and Biomimetic Polymer Brushes Prepared via Surface-Initiated Atom Transfer Radical Polymerization
}

\author{
Laurent Lavanant and Harm-Anton Klok ${ }^{\star}$
}

\begin{abstract}
Surface-initiated controlled radical polymerization is a powerful strategy to tailor the chemical and physical surface properties of materials. This article highlights recent work from the author's laboratory in which surfaceinitiated atom transfer radical polymerization is used to generate biofunctional and biomimetic surface coatings. Three examples will be discussed. The first two examples are based on the surface-initiated atom transfer radical polymerization of 2-hydroxyethyl methacrylate and (polyethylene glycol) methacrylate, which generates a polymer brush that suppresses non-specific adhesion of proteins and cells. These non-fouling brushes have been used to generate protein microarrays and to produce coatings that can promote endothelialization of implantable biomaterials. The third example describes the use of polyelectrolyte brushes as matrices to direct the mineralization of calcium carbonate.
\end{abstract}

Keywords: Biomaterials · Biomineralization · Polymer brushes · Protein microarrays . Surface-initiated polymerization

\section{Introduction}

The term polymer brush refers to a welldefined arrangement of polymer chains, which are tethered with one end to the surface of a solid substrate. ${ }^{[1,2]}$ At sufficiently high chain densities, due to steric repulsion, the polymer chains adopt a stretched chain conformation, which significantly differs from the random walk conformation of free polymer chains in solution or in conventionally solution-casted polymer films. ${ }^{[3]}$

Polymer brushes can be prepared either by the grafting-from or by the grafting-to approach (Fig. 1). The grafting-to strategy involves the covalent attachment of presynthesized polymers with an appropriate
${ }^{*}$ Correspondence: Prof. Dr. H.-A. Klok

École Polytechnique Fédérale de Lausanne (EPFL) Institut des Matériaux, Laboratoire des Polymères Bâtiment MXD, Station 12

$\mathrm{CH}-1015$ Lausanne

Tel.: + 41216934866

Fax: + 41216935650

E-mail: harm-anton.klok@epfl.ch reactive endgroup to a solid substrate functionalized with a complementary reactive group. The grafting-from strategy involves the preparation of polymer brushes from surfaces modified with appropriate initiating groups. From an experimental point of view, the grafting-to approach is advantageous as it is a single-step procedure and can readily be transferred to materials with complex shapes..$^{[4]}$ The grafting-to strategy, however, suffers from a number of drawbacks. ${ }^{[5]}$ First of all, grafting densities are limited due to steric crowding of already tethered polymer chains. Secondly, the film thicknesses are limited by the molecular weight of polymers in solution, which makes it difficult to prepare brushes with thicknesses in the $100 \mathrm{~nm}$ range. The grafting-from strategy, in contrast, does not suffer these limitations. Especially, when any of the nowadays manifold controlled/'living' polymerization methods is used, the grafting-from strategy allows the preparation of polymer brushes with a very high degree of control over brush thickness, composition, density and architecture. ${ }^{[6-10]}$ The graftingfrom strategy, which is also referred to as surface-initiated polymerization can also

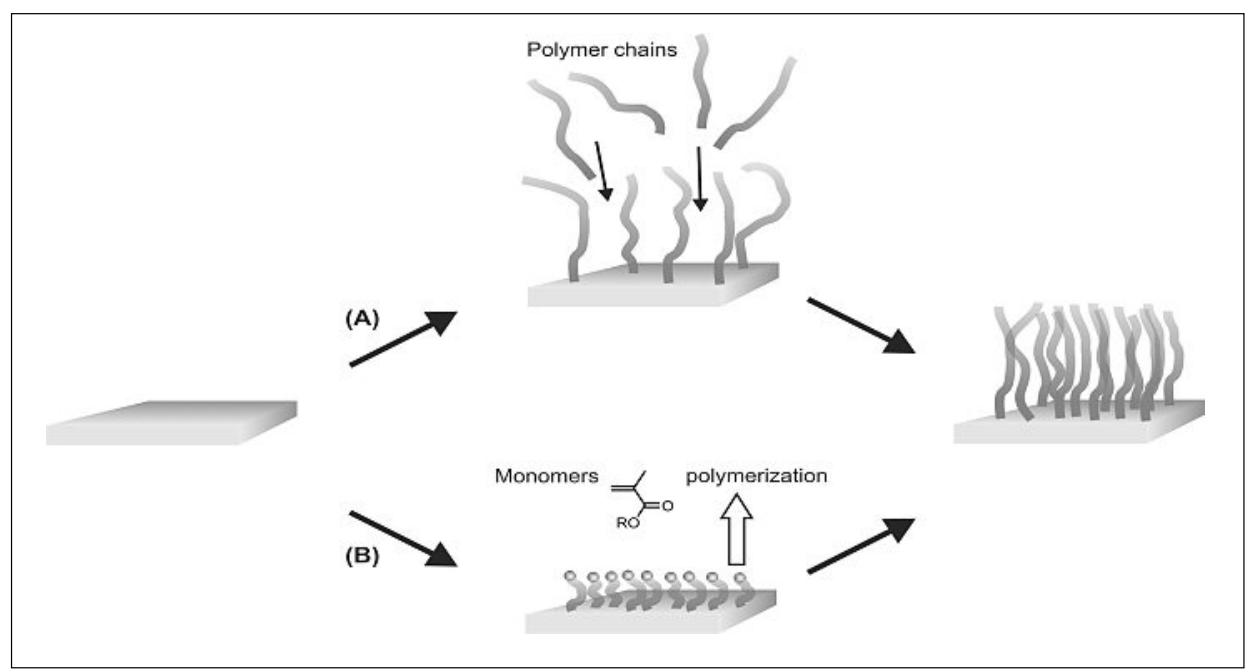

Fig. 1. Synthesis of polymer brushes: (A) grafting-to strategy; (B) grafting-from strategy 
easily be combined with various lithographic techniques, which allows additional control over the topography of surface-attached polymer brushes.

Polymer brushes prepared via surfaceinitiated polymerization have been explored for various applications. Surface-initiated atom transfer radical polymerization of 2-hydroxyethyl methacrylate (HEMA) ${ }^{[11]}$ (polyethylene glycol) methacrylate (PEGMA) ${ }^{[11-16]}$ and 2-methacryloyloxyethyl phosphorylcholine (MPC) ${ }^{[17]}$ have been used to generate non-fouling brushes that effectively resist non-specific protein and cell adhesion. The use of thermosensitive polymers such as poly(N-isopropylacrylamide) allows access to polymer brushes that can be reversibly switched from a hydrophilic, biologically inert state to a hydrophobic protein and cell adhesive state. [14] Polymer brushes prepared via the grafting-from approach have also been used to prepare antibacterial coatings ${ }^{[18]]}$ and have been proposed as nanoactuators. ${ }^{[19]}$ In addition to the modification of planar substrates, the grafting-from strategy has also been successfully used to modify the properties of porous alumina ${ }^{[20-22]}$ or polymer membranes. ${ }^{[23-25]}$

In most instances, the polymer brushes mentioned above were grown using standard copper-catalyzed procedures. Although the brushes are usually extensively washed after synthesis, there is a potential risk that some residual copper catalyst may remain. For the examples that will be discussed in this contribution, however, we are not aware of any adverse effects of possible catalyst residues on the properties of the polymer brushes. Furthermore, recent advances in atom transfer radical polymerization have resulted in initiator systems that reduce the amount of catalyst needed to ppm levels, where its removal may no longer be necessary for many applications. [26,27]

One of the interests of our laboratory is the use of surface-initiated atom transfer radical polymerization to produce ultrathin polymer coatings that endow the underlying substrate with specific biological properties or which can template the biomimetic formation of thin inorganic films. This article will give an overview of recent work from our laboratory in this area and will discuss three specific examples. As a first example, the use of non-fouling poly(poly(ethylene glycol) methacrylate) (PPEGMA) brushes as platforms for the fabrication of protein function microarrays will be presented. ${ }^{[28,29]}$ In the second example, it will be demonstrated that appropriately peptide-functionalized PPEGMA brushes are potentially interesting coatings to promote endothelialization of blood-contacting biomaterials. ${ }^{[30,31]}$ The third and last example will describe the use of photolithographically patterned polyelectrolyte brushes as biomi-

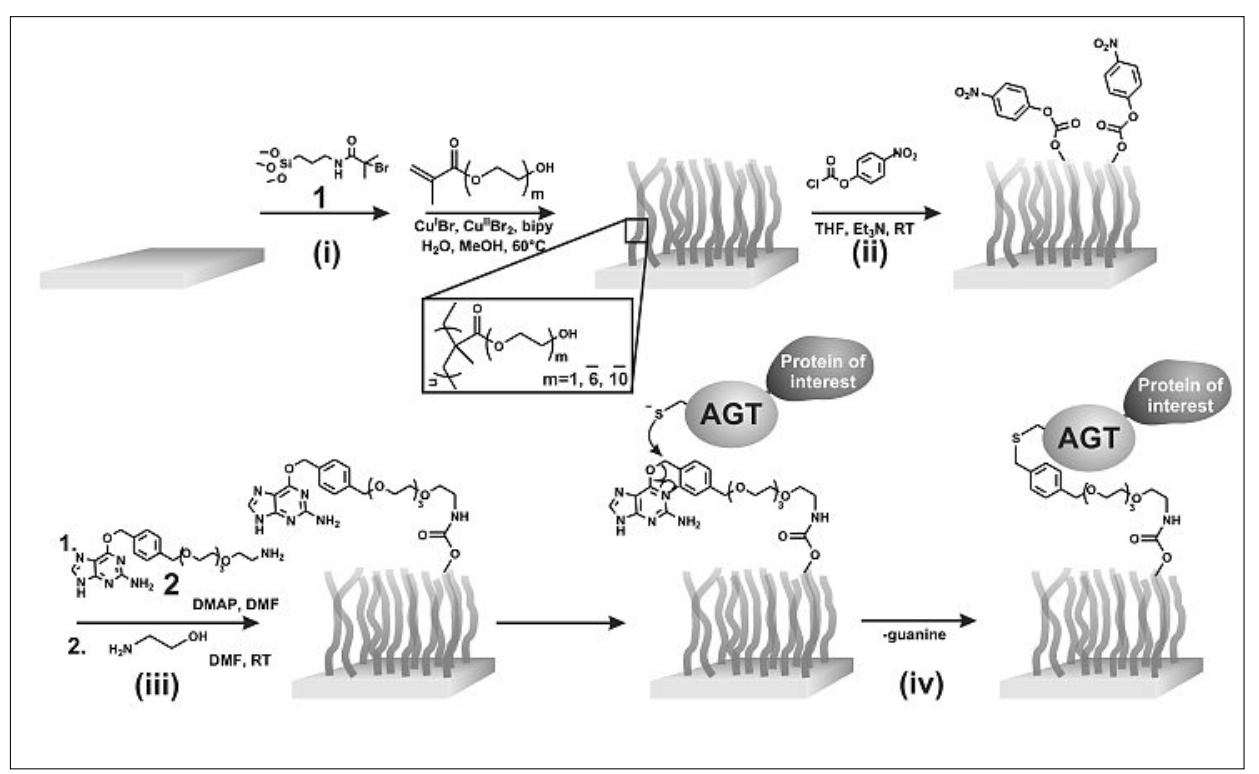

Fig. 2. Fabrication of protein functionalized polymer brushes: (i) grafting of polymerization initiator 1 and surface-initiated atom transfer radical polymerization, (ii) activation of hydroxyl groups with $p$-nitrophenyl chloroformate (NPC), (iii) functionalization with benzylguanine derivative $\mathbf{2}$ and quenching of residual NPC groups, (iv) immobilization of AGT fusion proteins on benzylguanine displaying surfaces

metic matrices to template the fabrication of thin microstructured calcite films. ${ }^{[32,33]}$

\section{Polymer Brushes for Protein Microarray Applications}

Protein microarrays consist of large numbers of proteins that are immobilized in defined patterns on a solid substrate. Protein microarrays are attractive tools for proteomics research as they enable highthroughput characterization of proteins. Proteins microarrays can be used both to measure the abundance of certain proteins as well as to study their function (i.e. interactions and biochemical activity).[34-36] In spite of the increasing interest in this class of bioanalytical devices, there is still a number of technological challenges that provides room for future improvement. Interesting challenges from a chemistry/materials perspective include the development of microarray surfaces with improved resistance toward non-specific protein adsorption and strategies that allow the chemoselective, covalent immobilization of proteins with retention of structure and function.

Fig. 2 illustrates an approach towards protein function microarrays that attempts to address both of the challenges mentioned above. The surface of the microarray chip is covered with a thin (20 to $200 \mathrm{~nm}$ ) poly (2hydroxyethyl methacrylate) (PHEMA) or PPEGMA brush, which serves two functions. First of all, the PHEMA and PPEGMA brush serves to suppress non-specific protein adsorption. Secondly, the hydroxyl groups, which are pendent to the polymer chains that constitute the polymer brush, provide anchoring points for the covalent immobilization of proteins. Protein functionalization following the strategy outlined in Fig. 2 involves the use of fusion proteins of a protein of interest with $\mathrm{O}^{6}$-alkylguanine-DNA-alkyltransferase (AGT) and takes advantages of the ability of AGT to transfer the alkyl group of $\mathrm{O}^{6}$ alkylguanine derivatives to one of its own cysteine residues. ${ }^{[37]}$ Amongst others, the AGT-mediated immobilization offers the following advantages: i) immobilization occurs exclusively via the AGT fusion and leaves the protein of interest accessible for interactions with other molecules, ii) functionalization is chemoselective and can be carried out directly from crude cell lysates without the need for purification steps.

The fabrication of the protein functionalized brushes outlined in Fig. 2 starts with the modification of a glass or silicon substrate with atom transfer radical polymerization initiator $\mathbf{1}$, which contains a bromoisobutyryl group. The bromoisobutyryl functionalized surfaces are subsequently used to initiate the atom transfer radical polymerization of HEMA or PEGMA. [28] Functionalization of the polymer brushes starts with activation of the hydroxyl groups with $p$-nitrophenyl chloroformate (NPC) in anhydrous tetrahydrofuran in the presence of triethylamine. ${ }^{[28]}$ After that, the activated brush coated substrate is derivatized with the benzylguanine functionalized amine $\mathbf{2}$ using a protein microarrayer. After a quenching step to remove any unreacted NPC groups, the modified surfaces are incubated in a solution containing the appropriate AGT fusion protein and finally washed to remove unbound protein. Fig. 
3 illustrates the immobilization of a Cy5 labelled fusion protein of AGT and dehydrofolate reductase (DHFR) on a PPEGMA brush following this process. The fluorescence image in Fig. 3 was obtained on a PPEGMA-coated surface of which only one part was modified with benzylguanine derivative 2 . The other three parts of this slide served as controls and were modified with benzylamine, 4-dimethylaminopyridine (DMAP) and N,N-dimethylformamide (DMF), respectively. The absence of fluorescence on these three parts of the substrate confirms the ability of the PPEGMA brush coating to suppress non-specific protein adsorption. Protein microarrays prepared according to the strategy outlined in Fig. 2 have been successfully used to measure protein heterodimerization, to detect protein-small molecule interactions and to monitor posttranslational modifications. ${ }^{[29]}$

\section{Polymer Brushes as Coatings for Blood-contacting Biomaterials}

An important goal in modern biomaterials research is the development of novel synthetic biomaterials, which are able to provide a microenvironment that mimics the natural extracellular matrix (ECM). ${ }^{[38,39]}$ Such materials are of great interest, not only for tissue engineering and regenerative medicine, but also for fundamental biological studies. Ideally, such biomaterials are intrinsically biologically inert, i.e. they resist the non-specific adhesion of proteins and cells, but offer possibilities to introduce specific peptide ligands. In this way, defined chemical cues can be presented to the environment. The most common peptide ligands that are used to create ECM mimetic microenvironments are based on the arginine-glycine-aspartic acid (RGD) sequence. ${ }^{[40]}$ This sequence is derived from the cell attachment domain of fibronectin and specifically binds to integrin receptors that are present on the cell surface. ${ }^{[41]} \mathrm{In}$ addition to biological non-fouling properties, it is also advantageous if the surface of the synthetic biomaterial can be structured with topographical cues with length scales that vary from several nanometers to a few micrometers. A number of strategies is available for the surface modification of biomaterials, including the use of self-assembled monolayers ${ }^{[6]}$ and plasma modification, ${ }^{[42]}$ amongst others. The use of surface-initiated controlled polymerization to modify biomaterials surfaces with a thin polymer brush coating is an interesting alternative, which offers good control over surface coverage, thickness and composition.

Due to their non-fouling properties and the presence of hydroxyl groups, which allow for post-polymerization modification with biologically active functional groups,

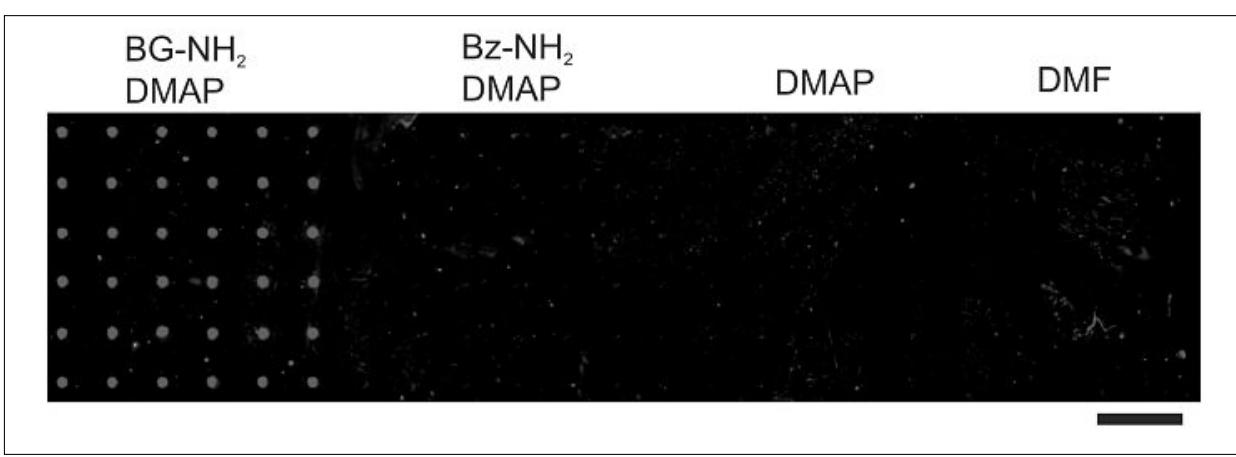

Fig. 3. Fluorescence image of a PPEGMA brush coated surface after NPC activation, spotting of $2 /$ DMAP, benzyl amine $\left(\mathrm{Bz}_{\mathrm{N}} \mathrm{NH}_{2}\right)$ /DMAP, DMAP in DMF and pure DMF (from left to right) followed by quenching and incubation with AGT-DHFR-MTX-Cy5 (scale bar: $1 \mathrm{~mm}$ )

the PHEMA and PPEGMA brushes discussed in the previous paragraph are of potential interest as biologically active coatings to modify biomaterials surfaces. To evaluate the feasibility of these brushes as bioactive biomaterials coatings, a series of PHEMA and PPEGMA brushes was grafted from glass substrates following the approach illustrated in Fig. 2. Modification of these polymer brushes with an RGDcontaining peptide as well as a scrambled RDG control sequence was achieved by NPC activation of the brush hydroxyl groups, followed by reaction with the appropriate peptide. The concentration of the peptide presented at the brush surface could be varied by adjusting the concentration of peptide in the reaction mixture during the

post-polymerization modification. ${ }^{[30]}$ Adhesion experiments with human umbilical vein endothelial cells (HUVECs) revealed the formation of confluent cell layers above a threshold RGD surface concentration of 1.0-5.3 pmol.cm ${ }^{-2}$. Fig. 4 compares HUVEC densities four hours postseeding on three polymer brush coatings, which were prepared via surface-initiated atom transfer radical polymerization of HEMA and two PEGMA derivatives with different ethylene glycol side chain lengths. For each brush, four different samples were investigated: i) neat (the polymer brush as obtained directly after polymerization); ii) quenched (the polymer brush after NPC activation and quenching with ethanolamine); iii) RGD functionalized; iv) RDG functionalized.

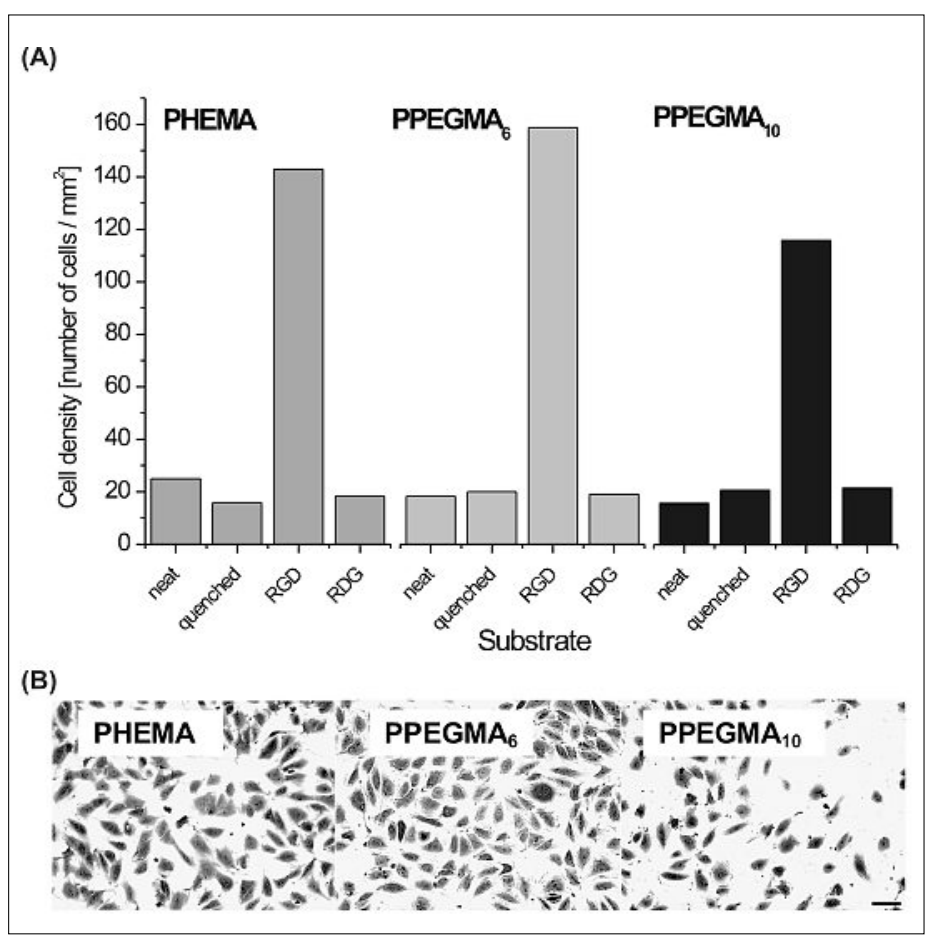

Fig. 4. (A) HUVEC densities $4 \mathrm{~h}$ postseeding on neat, quenched (NPC activated and ethanolamine treated), RGD and RDG functionalized 20 $\mathrm{nm}$ thick PHEMA, PPEGMA ${ }_{6}$ and PPEGMA 10 brushes supported on glass slides. (B) Photomicrographs of HUVECs $4 \mathrm{~h}$ postseeding on RGD functionalized $20 \mathrm{~nm}$ thick PHEMA, PPEGMA ${ }_{6}$ and PPEGMA 10 brushes. Peptide concentrations used for the functionalization were $1 \mathrm{mM}$. Scale bar: $50 \mu \mathrm{m}$. 
Fig. 4 clearly illustrates that cells adhere well to the RGD presenting brushes, whereas only low cell densities were observed on the control surfaces. This observation demonstrates that cell adhesion on the RGD functionalized surfaces is mediated by integrin-specific interactions and does not involve non-specific interactions.

At sufficiently high peptide surface concentrations, focal adhesions were established on all of the investigated polymer brush substrates.[30] This is illustrated in Fig. 5, which shows an overlay of the fluorescence micrographs of anti-vinculin (to visualize focal adhesions) and 4',6-diamidino-2-phenylindole (DAPI, to visualize the cell nucleus) stained HUVECs on RGDfunctionalized PHEMA and PPEGMA brush coatings. Interestingly, the morphology and size of the focal adhesions were different on the different brushes. Whereas HUVECs on PHEMA brushes established mainly large and mature focal adhesions at the cell periphery, smaller focal adhesions with fibrillar character were found in HUVECs that adhered to RGD-functionalized PPEGMA $_{6}$ or PPEGMA 10 brushes. These differences are not fully understood at the moment. There are, however, a number of factors that may contribute to these differences. First of all, as illustrated in Fig. 5B, there is about a factor of 2 difference in the peptide surface concentration between the PHEMA- and PPEGMA-based brushes. In both cases, however, the peptide concentrations were sufficient to allow focal contact formation. A second difference is related to the water solubility/swellability of the brushes. In contrast to PPEGMA, PHEMA does not dissolve but only swells in water.[43] Finally, the three polymer brushes differ with respect to the length of the ethylene glycol spacer that connects the RGD ligand to the polymer main chain. This spacer consists of a single ethylene glycol unit in the case of the PHEMA brushes and of, on average, six or ten ethylene glycol repeat units in the case of the PPEGMA brushes. An interesting hypothesis, which needs to be supported by additional data, would be that the enhanced water solubility of the PPEGMA brushes in combination with the increased ethylene glycol spacer length, leads to increased ligand mobility and reduced ligand-integrin affinity. HUVECs adhering to RGD functionalized PHEMA or PPEGMA brushes were able to withstand a fluid shear stress of $1.5 \mathrm{~kg} . \mathrm{m}^{-1} \cdot \mathrm{s}^{-2}$ for a period of 24 h. ${ }^{[30]}$ Fluorescence staining of the F-actin cytoskeleton revealed that the cells elongated in response to the shear stress and aligned parallel to the direction of the flow. As the shear stress that was used resembles the stress experienced by the endothelial cells that line the interior of blood vessels, these experiments suggest that PHEMA and PPEGMA brushes may be interesting

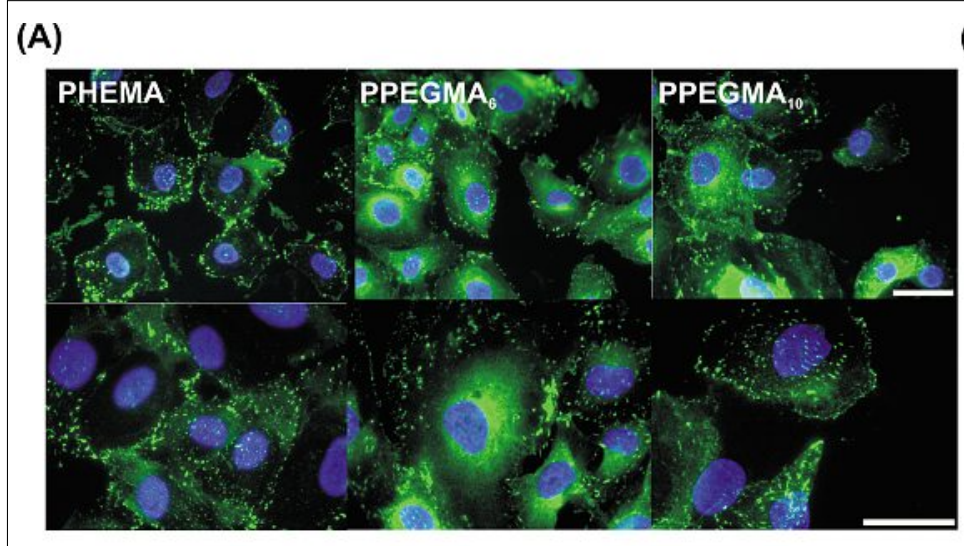

(B)

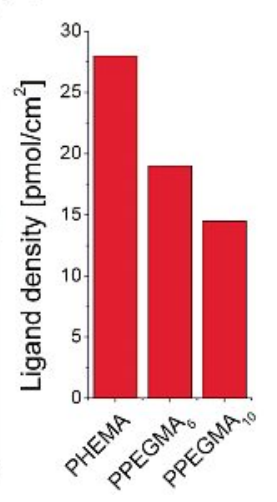

Fig. 5. (A) Overlay of fluorescence micrographs of HUVECs $4 \mathrm{~h}$ postseeding adhering to RGD functionalized $20 \mathrm{~nm}$ thick PHEMA, PPEGMA 6 and PPEGMA 10 brushes stained for nuclei and vinculin. The lower three micrographs were recorded with higher magnification. The RGD concentration for the functionalization was $1 \mathrm{mM}$. Scale bar: $25 \mu \mathrm{m}$; (B) RGD surface densities on PHEMA, PPEGMA 6 and PPEGMA 10 brushes functionalized using 1mM RGD and $2.5 \mathrm{mM}$ DMAP in DMF.

coatings to modify the surface properties of blood-contacting biomaterials.

\section{Polymer Brushes as lonotropic Matrices for the Fabrication of Microstructured Calcite Films}

Calcium carbonate is one of the most abundant biominerals and is widely used for the construction of the protective shells and casings of many organisms. ${ }^{[44]}$ Acidic matrix macromolecules have been identified to play an important role in guiding the mineralization of biogenic calcium carbonate. ${ }^{[45]}$ In addition to three crystalline polymorphs, biogenic calcium carbonate also occurs in two amorphous modifications: a stable hydrated form and a second less stable, essentially anhydrous form, which can act as a transient precursor for the thermodynamically stable crystalline modifications. ${ }^{[46]}$ The formation of various complex biogenic calcium carbonate structures has been proposed to proceed via the second, metastable amorphous calcium carbonate (ACC) phase. ${ }^{[47,48]}$ ACC as a transient precursor has been exploited in vitro to produce calcium carbonate crystals in constrained geometries, to fabricate microstructured calcite single crystals and to synthesize nacre-type laminated calcium carbonate coatings. [49-51] The attractiveness of the metastable ACC phase lies in the fact that it can be more easily molded into complex structures compared to the crystalline calcium carbonate polymorphs. In a subsequent step, the ACC phase can be converted into a crystalline phase by subjecting the material to brief thermal treatment. ${ }^{[51]}$ The application of such biological concepts to direct the size, shape and microstructure of synthetic inorganic materials is of great technological importance and could lead to new materials with potential applications in e.g. optical information processing, catalysis and regenerative medicine. [52-55]

It has been demonstrated that thin organic or polymer films are interesting tools to study and direct calcium carbonate mineralization. Substrates that have been used include acid functionalized self-assembled monolayers of alkanethiols on gold, ${ }^{[56]}$ Langmuir monolayers of acidic surfactants ${ }^{[57]}$ as well as polymeric LangmuirSchaefer films of acidic polydiacetylenes. ${ }^{[58]}$ Other approaches that have been explored include the use of immobilized organic/ polymer films, such as polyacrylic acid/ chitosan membranes, ${ }^{[59]}$ poly(vinyl alcohol), [60] anionic starburst dendrimers, ${ }^{[61]}$ collageneous matrices, ${ }^{[62]}$ and organosilane based thin films. ${ }^{[63]}$ These last five examples, however, involve the use of deposition techniques that usually only provide limited means of controlling lateral dimensions, surface patterning and thickness of the organic coating, which is often required for advanced technical applications.

An alternative approach to control the mineralization of calcium carbonate is outlined in Fig. 6 and is based on the use of negatively charged polyelectrolyte brushes, which are prepared via surface-initiated atom transfer radical polymerization of sodium methacrylate. ${ }^{[32,33]}$ The rationale behind the use of these negatively charged polymer brushes was that they could serve to mimic the negatively charged matrix macromolecules involved in calcium carbonate biomineralization and provide temporal stabilization for the transient ACC phase. In contrast to many of the approaches discussed in the previous paragraph, the thickness and composition of the polymer brush film can be accurately controlled using surface-initiated atom transfer radical polymerization. By combining this polymerization strategy with photolithographic techniques and the potential of negatively charged macromol- 
ecules to stabilize ACC, the objective of the approach outlined in Fig. 6 was to explore patterned polymer brushes as templates to guide the formation of microstructured, crystalline calcite films.

Microstructured poly(methacrylic acid) (PMAA) brushes were prepared from photolithographically patterned initiator-modified substrates. ${ }^{[32]}$ The patterned initiator functionalized substrates were obtained by UV-irradiation of silicon substrates modified with an atom transfer radical polymerization initiator-modified trimethoxysilane using a transmission electron microscopy grid as the photomask. This leads to decomposition of the initiator in the exposed regions, and subsequent polymerization of sodium methacrylate only takes place on regions of the substrate that are not irradiated.[17] As an example, Fig. 7 shows optical and atomic force microscopy (AFM) images of $90 \mathrm{~nm}$ thick PMAA brushes, which were prepared using photomasks of different mesh sizes.

Mineralization experiments were carried out by exposing glass substrates modified with the structured PMAA brushes to a continuous flow of a supersaturated calcium carbonate solution in a perfusion cell. After $30 \mathrm{~min}$, the samples were taken from the perfusion cell, washed, dried and analyzed with various microscopic techniques. Fig. 8A shows a differential interference contrast (DIC) micrograph of a 90 nm thick patterned PMAA brush after calcium carbonate mineralization. In a next step, the samples were subjected to a brief thermal treatment $\left(250{ }^{\circ} \mathrm{C}, 2 \mathrm{~h}\right)$ to convert the deposited ACC into a polycrystalline

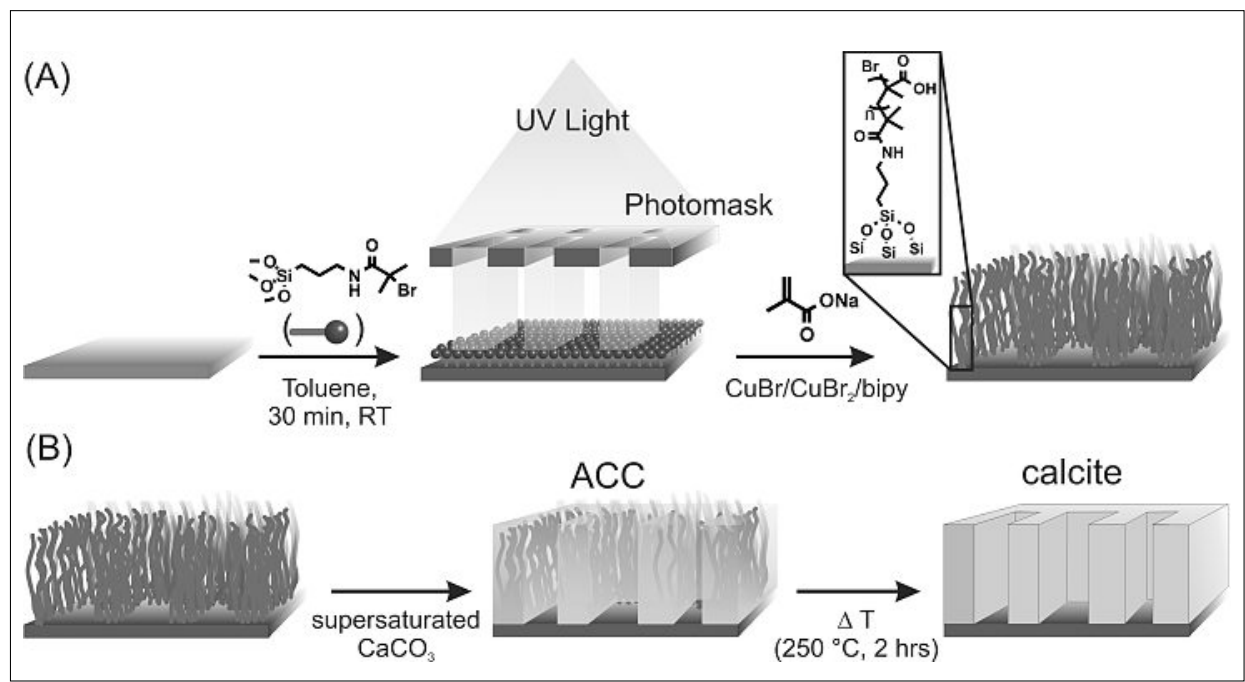

Fig. 6. Fabrication of microstructured calcite films. (A) Synthesis of microstructured poly(methacrylic acid) brushes via surface-initiated atom transfer radical polymerization of sodium methacrylate from photolithographically patterned initiator modified substrates. (B) Deposition of a thin, metastable layer of amorphous calcium carbonate (ACC) and subsequent temperature-induced transformation of the metastable ACC layer into a microstructured, polycrystalline calcite film.

calcite film. Comparison of Fig. 8A and $8 \mathrm{C}$ clearly indicates that deposition of ACC only occurs on regions of the substrate defined by the PMAA brush pattern. This point is particularly evident from the absence of birefringence in Fig. 8C in areas that are not covered with PMAA. Fig. $8 \mathrm{C}$ also shows that after thermal treatment, areas of the substrate that had been covered with PMAA reveal a mosaic-like birefringence texture, which indicates the formation of a crystalline calcite film. Using AFM, the thickness of the microstructured calcite film could be estimated at $~ 80-90 \mathrm{~nm}$. This suggests that mineralization of calcium carbonate occurs in the PMAA brushes and indicates that the polycrystalline calcite thin film is an exact 3D replica of the ionotropic PMAA matrix. While the lateral dimensions of the structured calcite films are defined by the photomask which is used to prepare the patterned substrates, the film thickness depends on the thickness of the PMAA brush, which can be controlled owing to the 'living'/controlled character of the SIATRP process.
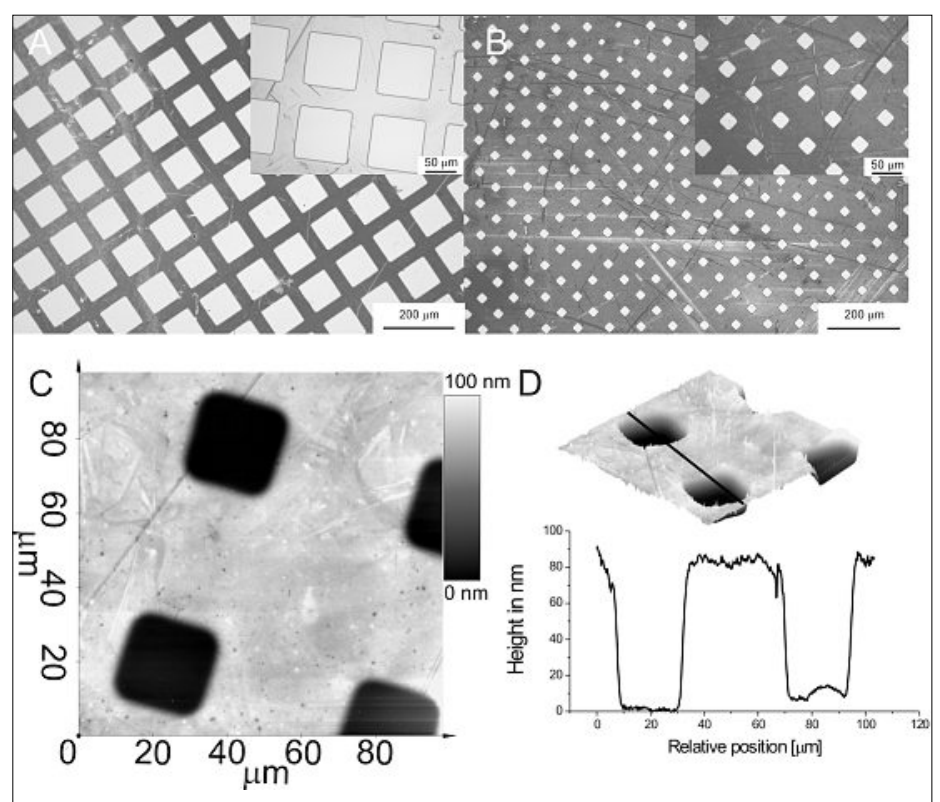

Fig. 7. (A),(B) Light microscopy images of photolithographically patterned PMAA brushes on silicon substrates prepared using different photomasks; (C) Topographical atomic force microscopy image and (D) 3D projection of the AFM image and height profile of a photolithographically patterned PMAA brush.

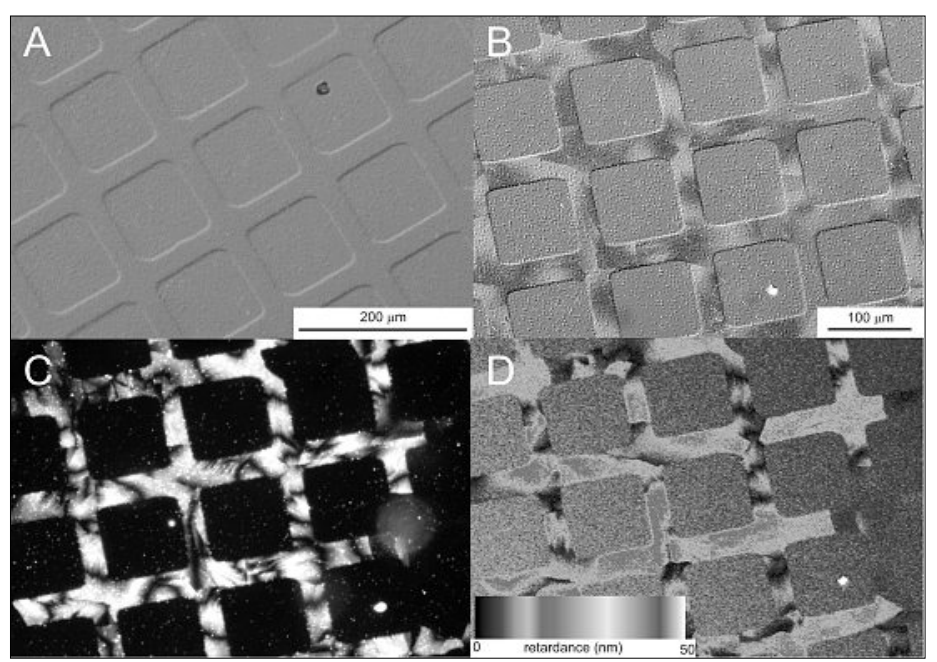

Fig. 8. (A) Differential interference contrast (DIC) micrograph of an ACC film deposited using a patterned $90 \mathrm{~nm}$ thick PMAA brush on a glass substrate; (B) Differential interference contrast; (C) polarized optical and (D) false color LC-PolScope images of the same calcium carbonate film after heating for $2 \mathrm{~h}$ at $250^{\circ} \mathrm{C}$. 


\section{Conclusions and Outlook}

Surface-initiated polymerization is a powerful method to modify the chemical and physical surface properties of materials. Over the past years, this technique has attracted the interest of an increasing number of research groups. In this article we have highlighted recent work from our own laboratory in which surface-initiated atom transfer radical polymerization is used to modify surfaces with ultrathin, functional polymer coatings. For additional examples and an overview of the excellent work performed by others active in this field, the reader is referred to several recent review articles. ${ }^{[6-10]}$ The examples discussed in this article demonstrate that surface-initiated atom transfer radical polymerization of 2-hydroxyethyl methacrylate (HEMA) or poly(ethylene glycol) methacrylate (PEGMA) is a powerful strategy to generate biologically inert (non-fouling) surfaces, which are attractive platforms to construct protein microarrays or biologically active biomaterial coatings that can promote endothelialization. In another example, it was shown that polyelectrolyte brushes generated from sodium methacrylate can act as three-dimensional matrices to direct the mineralization of calcium carbonate. This article only serves to give a first flavor of the possibilities offered by the use of surface-initiated controlled radical polymerization techniques to modify surface properties and generate functional surfaces. Although the power of this strategy has already been documented in a number of publications, its full potential has certainly not yet been explored. The continuous developments in the area of 'living'/controlled (radical) polymerization combined with innovative methods to immobilize polymerization initiators and the use of advance patterning techniques will undoubtedly lead to further applications of this strategy.

\section{Acknowledgements}

The authors wish to thank Prof. K. Johnsson (protein microarrays), Prof. N. Stergiopulos (cell adhesion), Prof. D. Volkmer (biomineralization) and their coworkers for their excellent collaboration, without which the work described in this article would not have been possible. Dr. S. Tugulu and R. Barbey from the author's laboratory are sincerely thanked for their contributions to the work described in this article. Our activities in this area are financially supported by the VolkswagenStiftung, the GEBERT RÜF STIFTUNG and CTI/KTI.

Received: May 22, 2008

[1] B. Zhao, W. J. Brittain, Prog. Polym. Sci. 2000, $25,677$.

[2] J. Rühe, W. Knoll, J. Macromol. Sci. Polym. Rev. 2002, C42, 91.

[3] S. T. Milner, Science 1991, 251, 905.
[4] A. Papra, A. Bernard, D. Juncker, N. B. Larsen, B. Michel, E. Delamarche, Langmuir 2001, 17, 4090.

[5] S. Edmondson, V. L. Osborne, W. T. S Huck, Chem. Soc. Rev. 2004, 33, 14.

[6] W. Senaratne, L. Andruzzi, C. K. Ober, Biomacromolecules 2005, 6, 2427.

[7] J.Pyun, T. Kowalewski, K. Matyjaszewski, Macromol. Rapid Commun. 2003, 24, 1043.

[8] G. K. Jennings, E. L. Brantley, Adv. Mater 2004, 16, 1983.

[9] Y. Tsujii, K. Ohno, S. Yamamoto, A. Goto, T. Fukuda, Adv. Polym. Sci. 2006, 197, 1.

[10] R. R. Bhat, M. R. Tomlinson, T. Wu, J. Genzer, Adv. Polym. Sci. 2006, 198, 51.

[11] R. R. Bhat, B. N. Chaney, J. Rowley, A. Liebmann-Vinson, J. Genzer, Adv. Mater. 2005, 17, 2802.

[12] X. Fan, L. Lin, J. L. Dalsin, P. B Messersmith, J. Am. Chem. Soc. 2005 , $127,15843$.

[13] X. Fan, L. Lin, P. B. Messersmith, Biomacromolecules 2006, 7, 2443.

[14] F. J. Xu, S. P. Zhong, L. Y. L. Yung, E. T. Kang, K. G. Neoh, Biomacromolecules 2004, 5, 2392.

[15] H. Ma, J. Hyun, P. Stiller, A. Chilkoti, Adv. Mater. 2004, 16, 338.

[16] F. J. Xu, Y. L. Li, E. T. Kang, K. G. Neoh, Biomacromolecules 2005, 6, 1759.

[17] R. Iwata, P. Suk-In, V. P. Hoven, A Takahara, K. Akiyoshi, Y. Iwasaki, Biomacromolecules 2004, 5, 2308.

[18] M. Ramstedt, N. Cheng, O. Azzaroni, D Mossialos, H. J. Mathieu, W. T. S. Huck, Langmuir 2007, 23, 3314.

[19] S. Moya, O. Azzaroni, T. Farhan, V. L. Osborne, W. T. S. Huck, Angew. Chem. Int. Ed. 2005, 44, 4578 .

[20] P. Jain, L. Sun, J. Dai, G. L. Baker, M. L. Bruening, Biomacromolecules 2007, 8 3102.

[21] L. Sun, J. Dai, G. L. Baker, M. L. Bruening, Chem. Mater. 2006, 18, 4033.

[22] L. Sun, G. L. Baker, M. L. Bruening, Macromolecules 2005, 38, 2307.

[23] N. Singh, S. M. Husson, B. Zdyrko, I. Luzinov, J. Membr. Sci. 2005, $262,81$.

[24] Z. Cheng, X. Zhu, E. T. Kang, K. G. Neoh, Macromolecules 2006, 39, 1660.

[25] F. J. Xu, J. P. Zhao, E. T. Kang, K. G. Neoh, J. Li, Langmuir 2007, 23, 8585.

[26] K. Matyjaszewski, W. Jakubowski, K. Min, W. Tang, J. Huang, W. A. Braunecker, N. V. Tsarevsky, Proc. Natl. Acad. Sci. USA 2006, 103, 15309.

[27] K. Matyjaszewski, H. Dong, W. Jakubowski, J. Pietrasik, A. Kusumo, Langmuir 2007, 23, 4528.

[28] S. Tugulu, A. Arnold, I. Sielaff, K. Johnsson, H.-A. Klok, Biomacromolecules 2005, 6, 1602 .

[29] I. Sielaff, A. Arnold, G. Godin, S. Tugulu, H.-A. Klok, K. Johnsson, ChemBioChem 2006, 7,194

[30] S. Tugulu, P. Silacci, N. Stergiopulos, H.A. Klok, Biomaterials 2007, 28, 2536

[31] S. Tugulu,H.-A.Klok, Biomacromolecules 2008, 9, 906.

[32] S. Tugulu, M. Harms, M. Fricke, D. Volkmer, H.-A. Klok, Angew. Chem., Int. Ed. 2006, 45, 7458.
[33] S. Tugulu, R. Barbey, M. Harms, M. Fricke, D. Volkmer, A. Rossi, H.-A. Klok Macromolecules 2007, 40, 168.

[34] B. Schweitzer, P. Predki, M. Snyder, Proteomics 2003, 3, 2190.

[35] T. Kodadek, Chem. Biol. 2001, 8, 105

[36] J. LaBaer, N. Ramachandran, Curr. Opin. Chem. Biol. 2005, 9, 14.

[37] A. E. Pegg, Mutat. Res. 2000, 462, 83.

[38] M. M. Stevens, J. H. George, Science 2005, 310, 1135 .

[39] M. P. Lutolf, J. A. Hubbell, Nat. Biotech. 2005, 23, 47 .

[40] U. Hersel, C. Dahmen, H. Kessler, Biomaterials 2003, 24, 4385 .

[41] E. Ruoslahti, M. D. Pierschbacher, Science 1987, 238, 491

[42] D. Klee, H. Höcker, Adv. Polym. Sci. 1999, $149,1$.

[43] J. V. M. Weaver, I. Bannister, K. L. Robinson, X. Bories-Azeau, S. P. Armes, M. Smallridge, P. McKenna, Macromolecules 2004, 37, 2395.

[44] B.-A. Gotliv, L. Addadi, S. Weiner, ChemBioChem 2003, 4, 522.

[45] S. I. Stupp, P. V. Braun, Science 1997, 277,1242

[46] L. Addadi, S. Raz, S. Weiner, Adv. Mater 2003, 15, 959 .

[47] Y. Politi, T. Arad, E. Klein, S. Weiner, L. Addadi, Science 2004, 306, 1161.

[48] N. Nassif, N. Pinna, N. Gehrke, M. Antonietti, C. Jäger, H. Cölfen, Proc. Natl. Acad. Sci. USA 2005, 102, 12653.

[49] E. Loste, F. C. Meldrum, Chem. Commun. 2001, 901 .

[50] J. Aizenberg, D. A. Muller, J. L. Grazul, D. R. Hamann, Science 2003, 299, 1205.

[51] D. Volkmer, M. Harms, L. Gower, A. Ziegler, Angew. Chem., Int. Ed. 2005, 44 , 639.

[52] D. Braga, Angew. Chem., Int. Ed. 2003 , 42,5544 .

[53] J. Aizenberg, Adv. Mater. 2004, 16, 1295.

[54] C. R. Martin, I. A. Aksay, J. Electroceram. 2004, 12, 53

[55] Y. Gao, K. Koumoto, Cryst. Growth Des. 2005, 5, 1983

[56] J. Aizenberg, A. J. Black, G. M Whitesides, Nature 1999, 398, 495.

[57] S. Mann, B. R. Heywood, S. Rajam, J. B. A. Walker, R. J. Davey, J. D. Birchall, $A d v$. Mater. 1990, 2, 257.

[58] A. Berman, D. J.Ahn, A.Lio, M. Salmeron, A. Reichert, D. Charych, Science $\mathbf{1 9 9 5}$ 269,515

[59] N. Wada, S. Suda, K. Kanamura, T. Umegaki, J. Colloid Interface Sci. 2004, $279,167$.

[60] N. Hosoda, A. Sugawara, T. Kato, Macromolecules 2003, 36, 6449 .

[61] Y. Tanaka, T. Nemoto, K. Naka, Y. Chujo, Polym. Bull. 2000, 45, 447.

[62] G. Falini, S. Fermani, M. Gazzano, A. Ripamonti, Chem. Eur. J. 1998, 4, 1048.

[63] D. D. Archibald, S. B. Qadri, B. P. Gaber, Langmuir 1996, 12, 538. 